\title{
Feasibility of home-based HIV counselling and testing and linking to HIV services among women delivering at home in Geita, Tanzania: a household longitudinal survey
}

\author{
Juma Adinan ${ }^{1,2,3^{*}}$ D, Bridgit Adamou ${ }^{4}$, Caroline Amour ${ }^{3}$, Aisa Shayo ${ }^{5}$, Paulo Lino Kidayi ${ }^{6}$ and Levina Msuya $a^{1,2,5}$
}

\begin{abstract}
Background: Substantial number of women who deliver at home (WDH) are not captured in prevention of mother-to-child transmission (PMTCT) services. This delays HIV infection detection that negatively impacts endeavours to fight the HIV pandemic and the health of mothers and children. The study objective was to determine the feasibility of home-based HIV testing and linking to care for HIV services among WDH in Geita District Council, Tanzania.
\end{abstract}

Methods: A longitudinal household survey was conducted. The study involved all mentally-able women who delivered within 2 years (WDTY) preceding the survey and their children under the age of two. The study was conducted in Geita District Council in Geita Region, Tanzania from June to July 2017. Geita is among the region with high HIV prevalence and proportion of women delivering at home.

Results: Of the 993 women who participated in the study, 981 (98.8\%) accepted household-based HIV counselling and testing (HBHCT) from the research team. HIV prevalence was 5.3\% (52 women). HBHCT identified 26 (2.7\%) new HIV infections; 23 (23.4\%) were those tested negative at ANC and the remaining three (0.3\%) were those who had no HIV test during the ANC visit. Among the $51 \mathrm{HIV}+$ women, 21 (40.4\%) were enrolled in PMTCT services. Of the $32 \mathrm{HIV}+$ participants who delivered at home, eight (25.8\%) were enrolled in the PMTCT compared to 100\% (13/13) of the women who delivered at a health facility.

Conclusion: HBHCT uptake was high. HBHCT detected new HIV infection among WDH as well as seroconversion among women with previously negative HIV tests. The study findings emphasize the importance of extending retesting to women who breastfeed. HBHCT is feasible and can be used to improve PMTCT services among WDH.

Keywords: PMTCT, Home-based HIV Counselling and testing, Linkage to care, Women delivering at home, Paediatric HIV, Home deliveries

\section{Background}

Despite major progress in implementing interventions for PMTCT in Sub-Saharan Africa, rates of new paediatric HIV infections remain unacceptably high, contributing to over $10 \%$ of new HIV infections globally and $15 \%$ of all HIV related mortality [1] .

\footnotetext{
* Correspondence: adinanjuma@gmail.com

${ }^{1}$ AMO School KCMC, P.O.Box 2316, Moshi, Tanzania

${ }^{2}$ Kilimanjaro Christian Medical Centre, Community Health department, Moshi, Tanzania

Full list of author information is available at the end of the article
}

The PMTCT program effectively reduces mother-tochild transmission of HIV. Women are enrolled in the program at the health facility, either at an antenatal care (ANC) visit, or when they come for delivery. In Tanzania, only around $60-70 \%$ of pregnant women receive HIV counselling and testing during ANC [2-5] and $49 \%$ of women do not deliver at a health facility [6]. Low testing uptake and home deliveries affects PMTCT enrolment and undermines the achievement of global HIV targets [7].

(c) The Author(s). 2019 Open Access This article is distributed under the terms of the Creative Commons Attribution 4.0 International License (http://creativecommons.org/licenses/by/4.0/), which permits unrestricted use, distribution, and reproduction in any medium, provided you give appropriate credit to the original author(s) and the source, provide a link to the Creative Commons license, and indicate if changes were made. The Creative Commons Public Domain Dedication waiver (http://creativecommons.org/publicdomain/zero/1.0/) applies to the data made available in this article, unless otherwise stated. 
Household-based HIV counselling and testing has been shown in different countries to be effective. $\mathrm{HBHCT}$ is an in-home HIV testing service where a person is referred to a health facility for subsequent care if s/he tests positive. Though there are documented drawbacks and conflicting results, HBHCT's effectiveness is widely accepted [8-14]. HBHCT has shown positive impact not only in detecting HIV new infections among pregnant and postpartum women [15] but also in influencing men to test [16-19] and participate in PMTCT services in Africa. Despite the introduction of home-based testing in Tanzania in 2013, HBHCT has never been evaluated in Tanzania for its effectiveness.

To achieve global HIV 90-90-90 [20] targets requires well-thought out and piloted strategies to detect HIV infection early and link HIV-positive individuals to care. Under the expanding PMTCT outcomes (EPO) project, we sought to determine the feasibility of home-based HIV testing and linking to HIV services among WDH in Geita District Council, Tanzania.

\section{Methods}

\section{Study design and setting}

The aim of the study was to determine the feasibility (i.e., perception plus uptake of HBHCT) of home-based HIV testing and linking to HIV services among WDH in Geita District Council, Tanzania. We employed longitudinal household survey conducted in Geita District Council, a rural area in Geita Region, Tanzania (Fig. 1). Three representative wards were selected: Nzera, Bugulula, and Rwamgasa. Socio-economic activities in Geita are mainly smallscale farming, business and mining. The study was conducted from June to July 2017.

In Geita Region, more women deliver at home (52\%) than the national average [21], but the adult HIV prevalence $(3-6 \%)$ is on par with the national average $(4.7 \%)$ [6].

\section{Sample size and sampling technique}

The study involved both WDH and women who delivered at a health facility within 2 years preceding the survey along with their children under the age of two. These two groups of women were compared in terms of acceptability and perception of household-based HIV counselling and testing (HBHCT). With no HIV prevalence data among WDTY in Geita District Council, the HIV prevalence of Geita Region was taken as a proxy estimate. We calculated a 5\% margin of error and $95 \%$ confidence interval, giving an initial sample size estimation of 844 . A standard $10 \%$ non-response rate was added to the sample to increase the minimum sample size to 928 WDTY. In total, 993 women participated in the study, along with 52 children whose mothers tested HIV-positive or refused an HIV test.

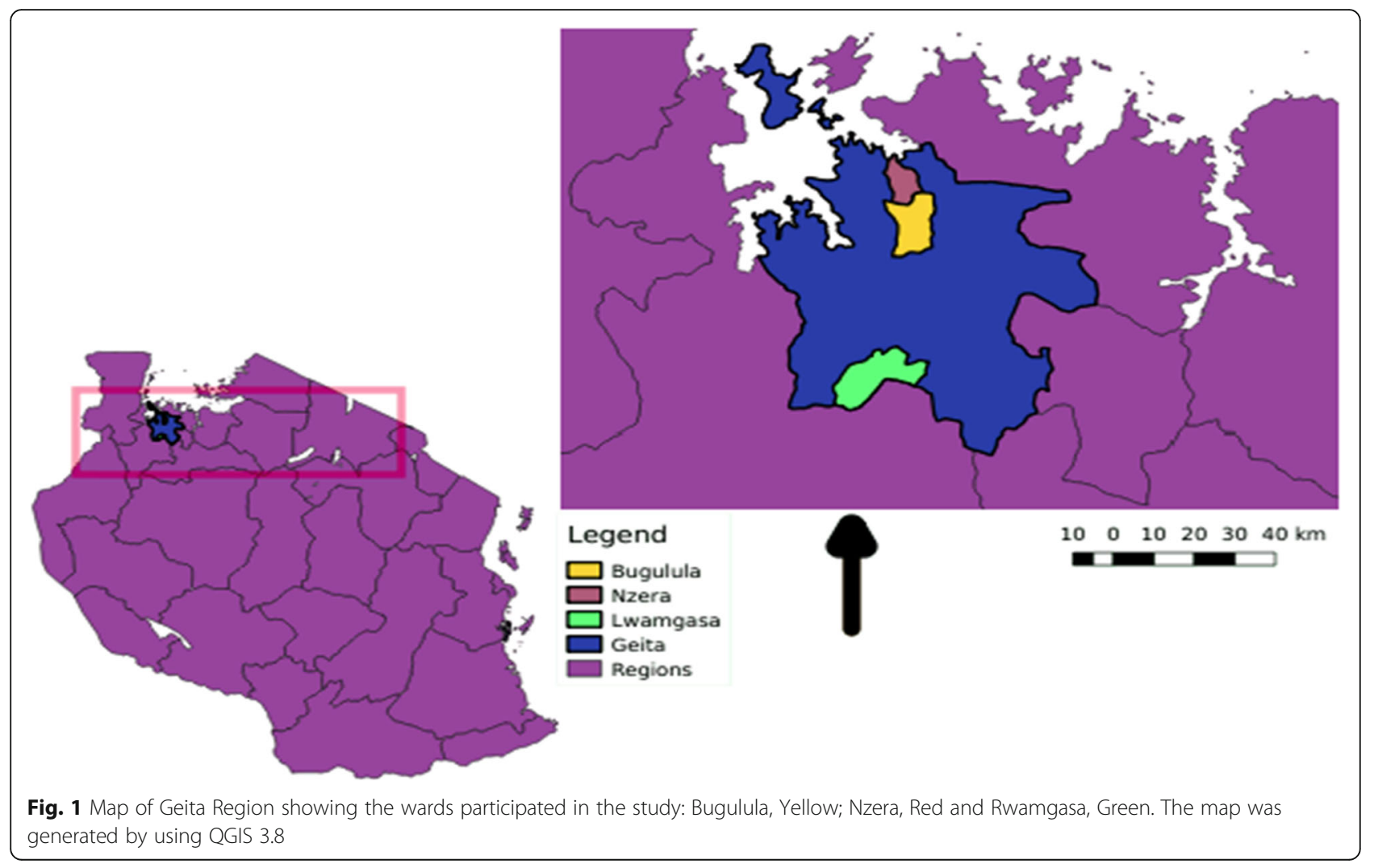




\section{Selection of households}

The unit of analysis was WDTY. A multistage cluster sampling technique was used to select households to be involved in the study. Three representative wards were selected: Nzera, Bugulula, and Rwamgasa. Within each ward, three villages were randomly selected and surveyed (except for Rwamgasa, where two villages were sampled). Two hamlets were selected from each village using simple random selection. The final stage was the systematic random selection of households. To identify households with WDTY, we asked household occupants. Once the household with WDTY was identified, we enrolled only one WDTY per household. If the household had more than one qualifying participant, we used random selection. A piece of paper was prepared based on the number of qualifying participants in the household. Each eligible individual randomly picked a piece of paper. Whoever picked the piece of paper that said "yes" was recruited into the study. Data collectors bypassed household without WDTY, they approached the subsequent house in the determined direction.

\section{Community sensitization}

To sensitize the community, we engaged local leaders in the process of implementing home-based HIV testing. The role of the local leaders were to inform the community of the project and to clear myths the community might have had about the goal of HIV testing.

Local leaders involved were ward executive officers from all participating wards, village chairmen and village secretaries from all participating villages.

We conducted a one-day workshop with the following objectives: 1) introduce the project and project team; 2) explain the importance of the project and findings; 3 ) describe how the project will be conducted; 4) explain the mobilization roles the leaders were going to play; and 4) share strategies of how to make the project successful based on people's experiences, for example, when to visit a house and other cultural matters.

Local leaders passed information to all households alerting the study population a day or two before we visited the household.

\section{Data sources, collection and referral Interview}

We used pretested questionnaire by Adeleke et al. [22]. The questionnaire contained structured and open-ended questions. Questions covered a range of topics including socio-demographic characteristics of the mothers and their children, mother's birth history which included ANC visits, PMTCT information (knowledge of PMTCT, history of HIV, number and interval of HIV tests done at ANC, and whether PMTCT services were provided), risk factors for acquiring HIV infection, household possessions for assessing wealth index, contextual factors that would influence healthcare-seeking behaviour, and questions related to HIV/ PMTCT program interventions. We collected qualitative data to gain an in-depth understanding of the reasons for not having an HIV test during ANC and reasons for not enrolling in PMTCT for the mothers who were aware of their HIV-positive status. Along with participant's contacts, we collected three contacts of participants' close people (relatives, friends or colleagues). These contacts were used to trace participants in case a break of communication between investigators and participant occurred.

\section{Home-based HIV counselling and testing}

Antibody-based HIV (HIV rapid) tests were used to determine the HIV-serostatus of study participants [23, 24]. HBHCT was offered to WDTY regardless of the time lapse since their last test. HIV tests were offered to those who consented to testing and to the children ages 9-23 months whose mothers tested HIV-positive as part of the study-administered HBHCT. We followed both the national HIV algorithm [25] and manufacturer directives for testing and results interpretation.

\section{Referral and follow-up phone interview}

We referred to a health facility for testing, care, and treatment, as necessary, mothers who had never tested for HIV previously and refused a study-administered HIV test; mothers who knew their HIV-positive status but were not enrolled in PMTCT; and mothers who were identified as HIV-positive by the study. Participants who were referred to a health facility for subsequent care were followed-up (Fig. 2) by phone three times (at 1 month, 2 months, and 3 months) to assess if they followed through on their referral, their HIV serostatus, and, if appropriate, uptake of PMTCT services. A successful referral was assessed at the last follow-up.

\section{Definition of variables}

Feasibility of HBHCT in detecting HIV was defined as an acceptance rate of $\geq 90 \%$ of home-based HIV testing and an acceptance rate of $\geq 90 \%$ of receiving results by women delivering at home/ within 2 years.

Feasibility in linking to care was defined as $\geq 50 \%$ of the referred individuals reporting a completed referral.

\section{Data quality assurance and analysis}

We used Kobotoolbox ${ }^{\bullet}$ for data collection. The study's primary outcome was the feasibility of HBHCT. The secondary outcome was the successful link to care of HIV positive WDH.

We transferred data from Kobotoolbox ${ }^{\circ}$ to Stata Version 15 for cleaning, processing, and analysis. The analysis took into consideration the clustering effect. 


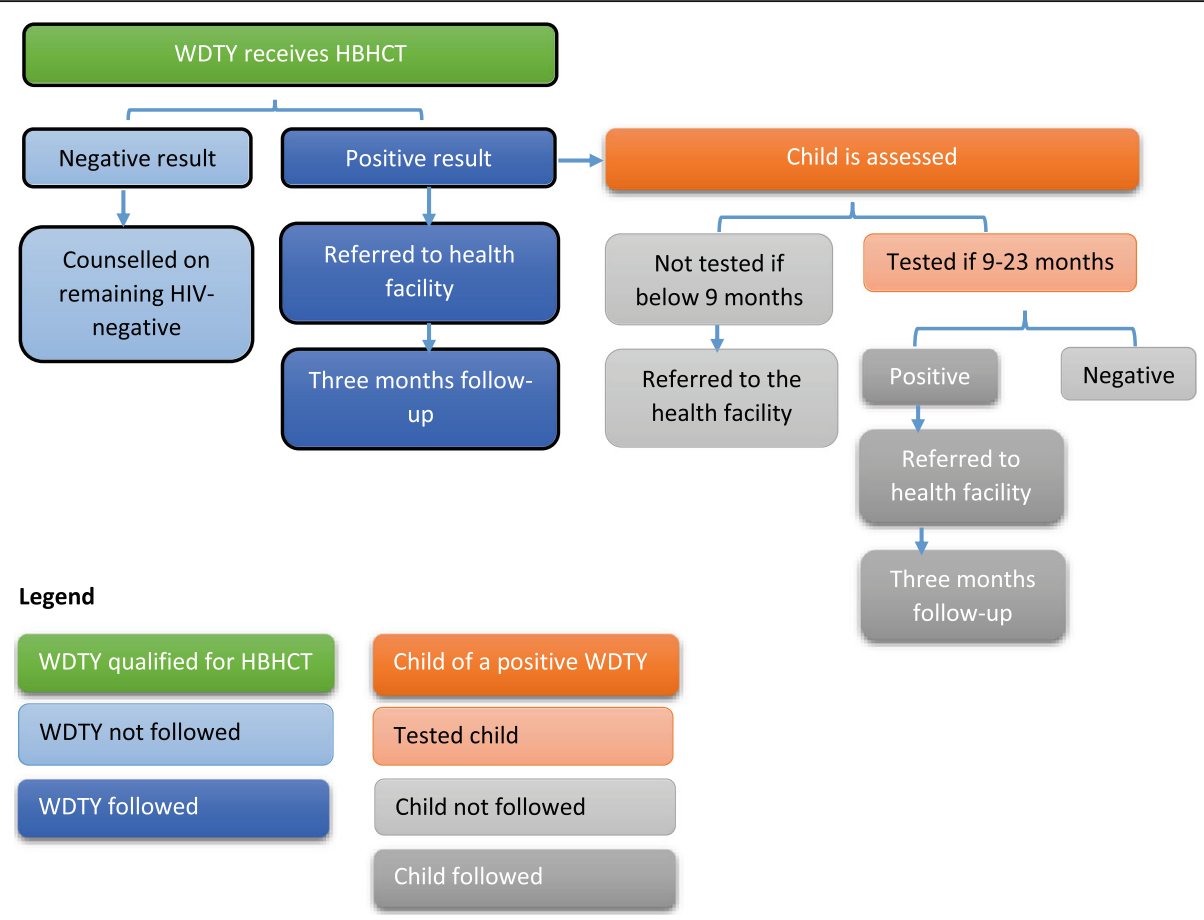

Fig. 2 Study Algorithm showing eligible participants and their level of participation into the study

Hamlets were clusters in this study. We used population statistics to generate post-sampling weight to account for the ward-level variance in sample size.

A $p$-value of less than 0.05 was considered for statistical significance independence during comparison between WDH and those delivered at the health facilities.

\section{Results}

\section{Background characteristics of participants}

In total, 993 WDTY in Geita District Council participated in the study. The median age was 25 years, with the largest proportion in the 16- to 24-year age band. Most resided in Rwamgasa (47.7\%), had a primary education (39.6\%), were married or in-union (89.1\%), and had one or two children (43.6\%). Fewer than $10 \%$ had health insurance coverage, and most (92.5\%) did not consider the health facility they usually access for health services as being far from their home. Nearly all the participants $(946 ; 95.2 \%)$ attended at least one ANC visit during their last pregnancy. Among these women, $81.7 \%$ (773) were tested for HIV during ANC attendance, with 26 (3.3\%) testing positive. More than half (56.9\%) of the participants had a home birth for their last delivery (Table 1).

\section{Uptake of HIV testing services at ANC and HBHCT}

Of the 946 (95.2\%) participants who visited ANC when pregnant, 767 (81.1\%) were tested for HIV. Of those tested, 26 (3.4\%) tested positive for HIV.
Nearly all the study participants (981; 99.8\%) accepted HBHCT. Specifically, 562 (99.4\%) of WDH and 419 (97.9\%) of women who delivered at a hospital accepted HBHCT. HBHCT identified 26 (2.7\%) new HIV infections among the women; 23 were those who had tested negative at ANC and the remaining three were those who had no HIV test during an ANC visit.

Reasons mentioned for not testing for HIV at an ANC visit included lack of testing kits at the ANC, not being given test results on time, and financial barriers. Reasons for refusing $\mathrm{HBHCT}$ included being recently tested for $\mathrm{HIV}$, needing permission from husband, and afraid of receiving a positive test result.

\section{Prevalence of HIV among participants}

The prevalence of HIV among WDTY was 52 (5.3\%) [95\% CI: $2.1-12.8 \%$ ]. Thirty one (59.6\%) of the HIVpositive participants had delivered at home; among the HIV-positive WDH, 18 (58.1\%) were identified during HBHCT. Most of these women were in the 25- to 35years age category (Fig. 3.).

Twenty-seven of the children delivered by HIVpositive women were tested for HIV. Seven children were HIV-positive. All were children of mothers who were newly detected by HBHCT; six were children of six mothers who had tested negative at ANC, and the one remaining was the child from the mother who had not had HIV test during ANC visit. 
Table 1 Participants' background characteristics ( $n=993)$. Un weighted and weighted Per cent distribution of participant's background characteristics, EPO project 2017

\begin{tabular}{|c|c|c|c|c|}
\hline & \multicolumn{2}{|c|}{ Not weighted } & \multicolumn{2}{|c|}{ Weighted } \\
\hline & No. & $\%$ & No. & $\%$ \\
\hline \multicolumn{5}{|l|}{ Age } \\
\hline $16-24$ & 435 & 43.80 & $478 / 479$ & 48.20 \\
\hline $25-35$ & 413 & 41.60 & 374 & 37.70 \\
\hline $36-51$ & 145 & 14.60 & 140 & 14.10 \\
\hline \multicolumn{5}{|l|}{ Education $^{\text {a }}$} \\
\hline No formal education & 342 & 34.50 & 324 & 32.60 \\
\hline Did not complete primary school & 206 & 20.80 & 217 & 21.80 \\
\hline Primary education & 402 & 40.50 & 393 & 39.60 \\
\hline Secondary education & 41 & 4.10 & 54 & 5.40 \\
\hline Higher education & 1 & 0.10 & 5 & 0.50 \\
\hline \multicolumn{5}{|l|}{ Marital status ${ }^{a}$} \\
\hline Single & 49 & 5.00 & 46 & 4.70 \\
\hline Married/In union & 851 & 87.10 & 870 & 89.10 \\
\hline Divorced & 77 & 7.90 & 61 & 6.20 \\
\hline \multicolumn{5}{|l|}{ Ward of residence } \\
\hline Nzera & 439 & 44.20 & 223 & 22.50 \\
\hline Bugulula & 397 & 40.00 & 296 & 29.80 \\
\hline Rwamgasa & 157 & 15.80 & $473 / 474$ & 47.70 \\
\hline \multicolumn{5}{|l|}{ Occupation } \\
\hline Small-scale farmers & 959 & 96.58 & 954 & 96.06 \\
\hline Self-employed business & 29 & 2.92 & 36 & 3.60 \\
\hline Salaried employee & 5 & 0.50 & 3 & 0.34 \\
\hline \multicolumn{5}{|l|}{ Number of children } \\
\hline $1-2$ & 397 & 40.00 & 433 & 43.60 \\
\hline $3-4$ & 264 & 26.60 & 262 & 26.40 \\
\hline $5-14$ & 332 & 33.40 & 298 & 30.00 \\
\hline \multicolumn{5}{|l|}{ Covered by health insurance ${ }^{a}$} \\
\hline No & 895 & 90.20 & 897 & 90.40 \\
\hline Yes & 97 & 9.80 & 95 & 9.60 \\
\hline \multicolumn{5}{|l|}{ Is the usual health facility you access far? } \\
\hline No & 858 & 86.40 & 918 & 92.50 \\
\hline Yes & 135 & 13.60 & 75 & 7.50 \\
\hline \multicolumn{5}{|l|}{ Place of last delivery } \\
\hline Home & 554 & 55.80 & 565 & 56.90 \\
\hline Health facility & 439 & 44.20 & 428 & 43.10 \\
\hline \multicolumn{5}{|l|}{ Attended ANC in the last pregnancy } \\
\hline No & 36 & 3.60 & 47 & 4.80 \\
\hline Yes & 957 & 96.40 & 946 & 95.20 \\
\hline \multicolumn{5}{|l|}{ HIV test at ANC visit $(n=957 / 946)$} \\
\hline No & 228 & 23.80 & 173 & 18.30 \\
\hline Yes & 729 & 76.20 & 773 & 81.70 \\
\hline
\end{tabular}

Table 1 Participants' background characteristics $(n=993)$. Un weighted and weighted Per cent distribution of participant's background characteristics, EPO project 2017 (Continued)

\begin{tabular}{|c|c|c|c|c|}
\hline & \multicolumn{2}{|c|}{ Not weighted } & \multicolumn{2}{|c|}{ Weighted } \\
\hline & No. & $\%$ & No. & $\%$ \\
\hline \multicolumn{5}{|c|}{ HIV results at ANC visit $(n=729 / 773)^{a}$} \\
\hline Positive & 12 & 1.65 & 26 & 3.34 \\
\hline Negative & 708 & 97.12 & 741 & 95.87 \\
\hline
\end{tabular}

Twenty-five children of HIV-positive mothers could not be tested as 24 were below 9 months of age and one was because of mother's refusal.

\section{PMTCT uptake among HIV-positive participants}

Among the $52 \mathrm{HIV}$-positive women, 21 were enrolled in PMTCT services. All 13 HIV-positive women who delivered at a health facility and knew their HIV-positive status before the HBHCT were enrolled in PMTCT services, contrary to their $13 \mathrm{WDH}$ counterparts, where only eight were enrolled in PMTCT services (Fig. 3.)

The five WDH who knew their HIV-positive status before the study mentioned fear of family conflicts as the main reason not to enrol in PMTCT services.

\section{Perception of effectiveness of HBHCT among participants} When participants were asked about their perception of HBHTC services compared to facility-based HIV testing and counselling, most agreed that HBHCT saves time (77.2\%), reaches more people (66.7\%), and links to PMTCT earlier (59.7\%). The differences in perception between Women delivering at facilities and women delivered at home that HBHCT saves time $(81.3 \% \mathrm{Vs}$ $77.2 \%$ ) and links to PMTCT earlier (64.5\% Vs 69.7\%) is statistically significant. (Table 2).

\section{Referrals to health facilities for HIV testing and PMTCT services}

The study team referred 43 mothers to health facilities; 12 for testing after denying HBHCT and 31 for subsequent PMTCT services following positive HIV results. Researchers managed follow-up communication with 16 participants and of these, 12 completed the referral. Almost two-thirds $(27 ; 62.8 \%)$ of the referred participants were not reachable for different reasons. Of the 12 participants who attended referral, seven were among those referred for testing.

Among the 31 participants who were HIV-positive and referred for subsequent PMTCT care, only five successfully attended referral; of these, four were newly-identified HIV-positive. Two participants gave the excuse that they were travelling and one reported lack of time. 


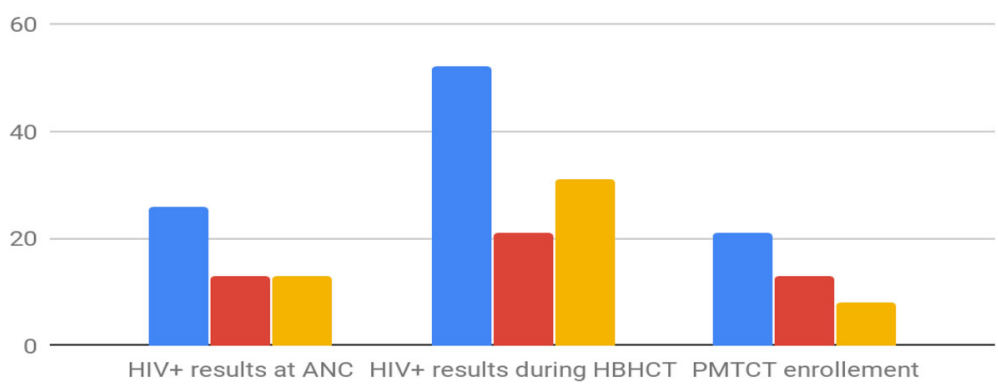

Fig. 3 Per cent distribution of HIV prevalence and uptake of PMTCT by places of delivery

\section{Discussion}

This study was conducted to determine the feasibility of HBHCT for early detection of HIV infection among women up to 2 years postpartum and linking HIVpositive women to subsequent HIV care in Geita District Council, Geita, Tanzania.

The HIV prevalence among the adult study participants was $5 \%$. This prevalence is similar to the HIV prevalence of 3-6\% reported by the National Bureau of Statistics (2016) [21]. The most affected were in the 25to 35-years age category and were residents of Rwamgasa-the ward where mining is the main economic activity. These support findings already reported on risky population groups based on the 25- to 35-years age [21] category living in areas where the main economic activity is mining [26].

The study showed that HBHCT is highly acceptable, with $99 \%$ of participants agreeing to be tested. The high acceptability signifies the suitability of HBHCT as a method of early HIV detection among the study population. This finding is similar to those reported in different parts of Africa [11, 27], including Tanzania [12, 28]. However, this study reported very high acceptability of HBHCT compared to other studies, which reported 60 $70 \%$ acceptance.

High acceptability can be explained by the fact that participants were reached and tested at their homes. The reason we had an increased uptake in the number of women to be tested was due to the fact that the women in this community are predominantly farmers. Hence most of their activities are being done near their homes, hence bringing the service close to home was readily accepted as it does not take them away from their activities. Also the men had a buyin in acceptance of the testing and since it is a predominantly male dominated community, we had a clear passage and an increased uptake in testing.

High acceptability can also be explained by the role played by local leaders in informing the community of the study and the trust that was built with the researchers. Local leaders did community mobilization on the screening services as well as escorting data collectors

Table 2 Perception of participants on HBHTC effectiveness, efficiency, and early care for HIV ( $n=933)$

\begin{tabular}{|c|c|c|c|c|}
\hline \multirow[t]{2}{*}{ Variables } & Facility-based delivery & Home-based delivery & Total & \multirow{2}{*}{$\begin{array}{l}\mathrm{X}^{2} \\
(P \text {-Values })\end{array}$} \\
\hline & No. $(\%)$ & No. (\%) & No. (\%) & \\
\hline \multicolumn{5}{|c|}{ HBHCT saves time } \\
\hline Agree & $357(81.3)$ & $410(74)$ & $767(77.2)$ & \multirow[t]{3}{*}{$7.80(0.020)$} \\
\hline Indifferent & $52(11.8)$ & $97(17.5)$ & $149(15)$ & \\
\hline Disagree & $30(6.8)$ & $47(8.5)$ & $77(7.8)$ & \\
\hline \multicolumn{5}{|c|}{ HBHCT reaches more people } \\
\hline Agree & $305(69.5)$ & $357(64.4)$ & $662(66.7)$ & \\
\hline Indifferent & $48(10.9)$ & $78(14.1)$ & $126(12.7)$ & \multirow[t]{2}{*}{$3.26(0.195)$} \\
\hline Disagree & 86 (19.6) & $119(21.5)$ & 205 (20.6) & \\
\hline \multicolumn{5}{|c|}{ HBHCT links to PMTCT earlier } \\
\hline Agree & $283(64.5)$ & $310(56)$ & $593(59.7)$ & \multirow{3}{*}{$7.65(0.022)$} \\
\hline Indifferent & $138(31.4)$ & $220(39.7)$ & $358(36.1)$ & \\
\hline Disagree & $18(4.1)$ & $24(4.3)$ & $42(4.2)$ & \\
\hline
\end{tabular}

Per cent distribution and comparison of perception of women who delivered at home versus those delivered at the health facilities on the importance of HBHCT in terms of saving time, coverage and link to PMTCT services earlier, EPO project 2017 
to households. Research assistants reported community members' readiness and enthusiasm towards HIV testing regardless of their eligibility criteria. This was evident by the 393 men and other women who did not meet the inclusion criteria who asked for the HIV test.

HBHCT identified half (50\%) of the HIV cases in our study population. More so, the study revealed seroconversion among women who tested negative during their last HIV test at ANC. These findings not only emphasize the importance of HBHCT in improving access to HIV services [8] but also the importance of extending retesting $[29,30]$ to women who breastfeed to effectively implement PMTCT programs. Seroconversion among pregnant women have previously been reported [31, 32]. These findings are similar to those reported in Malawi [12] and Tanzania [30].

Only about two-thirds (8/13) of the WDH who knew their positive HIV status before HBHCT were enrolled in PMTCT services compared to $100 \%(13 / 13)$ of the women who delivered at a health facility. These findings emphasize the importance of health facility deliveries over home deliveries and the importance of scaling up PMTCT services to women who deliver at home.

The strength of the study lies in the rigorous planning, data collection, and analysis. The study was able to show the feasibility of HBHCT in terms of acceptability and uptake among women who are within 2 years postpartum. The study managed to detect the majority of HIVpositive WDH who were unaware of their serostatus and linked them to PMTCT services.

However, the study's limitations are important to be noted. The study could not detect the effectiveness of HBHCT in linking HIV-positive women to PMTCT and subsequent HIV care. Also, the study suffered from communication barrier challenges that caused loss to follow-up. We used mobile phones to track participants and could only contact less than half of the referred participants. We could not test children younger than 9 months as we used antibody-based HIV rapid test. In addition to the limitation in the designing, HBHCT was offered to any women delivering at home within 2 years. So, some children might not have benefited from the intervention as the time for the PMTCT initiation might have had passed. Finally, we studied a rural population, so the study findings cannot be representative of an urban population.

These findings are important to policy makers and other stakeholders working to address HIV burden. With the increased evidence of higher risk of infection among pregnant and postpartum women [33] and poor implementation of repeat HIV testing during pregnancy $[34,35]$, the findings show the feasibility [36] and costeffectiveness [18] of home-based HIV counselling and testing. Beneficiaries of this study are national and sub- national stakeholders working to control and eradicate HIV, such as Ministries responsible for health, districtlevel health managers, and implementing partners; researchers in the field of community-based services, HIV, and health systems; and donors of HIV interventions.

\section{Conclusion}

HBHCT uptake was high. HBHCT detected new HIV infection among WDH as well as seroconversion among women with previously negative HIV tests. The study findings emphasize the importance of extending retesting to women who breastfeed. HBHCT can be used to improve PMTCT services among WDH.

\section{Abbreviations}

ANC: Antenatal care; HBHCT: Home-based HIV counselling and testing; HIV: Human immune deficiency virus; PMTCT: Prevention of mother to child transmission; WDH: Women who delivered at home; WDTY: Women who delivered within 2 years

\section{Acknowledgements}

We are grateful for the project's technical support from the review of the application to the write-up of the project report. We thank the knowledge management team of MEASURE Evaluation, University of North Carolina at Chapel Hill (USA) for editorial and production services.

We would also like to thank the following for their contributions: - Geita District Medical Officer and Assistant Medical Officers School Kilimanjaro Christian Medical Centre (KCMC) for providing logistical support - Geita local authority for granting permission to conduct the study - Geita District Council ward and village leaders for their full participation in the project and assistance with logistics and community sensitization - Study participants for their time and involvement

- Geita School of Nursing and Midwifery for providing supervisors and research assistants

- Research assistants for their involvement in data collection

\section{Authors' contributions}

$J A, P L K, C A$ and LM, performed the research. JA, CA, LM and PLK designed the research study. JA analyzed the data. JA, BA, CA, AS, PLK and LM. wrote the paper. All authors have read and approved the final manuscript.

\section{Funding}

The United States Agency for International Development (USAID) and the United States President's Emergency Plan for AIDS Relief (PEPFAR) funded this study through the MEASURE Evaluation-Tanzania Associate Award (Reference Number: 17628-KCMC-01). The funder played no role in the design of the study and collection, analysis, and interpretation of data and in writing the manuscript.

\section{Availability of data and materials}

The datasets analysed during the current study are available from the corresponding author on reasonable request.

\section{Ethics approval and consent to participate}

Ethical clearance to conduct the study was obtained from Kilimanjaro Christian Medical University College Clinical Research Ethics Review

Committee (Certificate No. 2005). Researchers also received permission from Geita District Council Authority to conduct the study.

The study participants provided signed informed consent prior to the data collection. A unique identifier was used to de-identify participants for confidentiality. Trained research assistants provided counselling to the study participants before and after administering HIV tests.

Consent for publication

Not applicable. 


\section{Competing interests}

The authors declare that they have no competing interests.

\section{Author details}

'AMO School KCMC, P.O.Box 2316, Moshi, Tanzania. ${ }^{2}$ Kilimanjaro Christian Medical Centre, Community Health department, Moshi, Tanzania. ${ }^{3}$ Kilimanjaro Christian Medical University College, Institute of Public Health, Moshi, Tanzania. ${ }^{4}$ University of North Carolina at Chapel Hill, Chapel Hill, USA. ${ }^{5}$ Kilimanjaro Christian Medical Centre, Paediatric and Child Health department, Moshi, Tanzania. ${ }^{6}$ Kilimanjaro Christian Medical University College, Faculty of Nursing, Moshi, Tanzania.

Received: 10 July 2019 Accepted: 19 December 2019 Published online: 30 December 2019

\section{References}

1. UNICEF. Paediatric Care and Treatment - UNICEF DATA, https://data.unicef. org/topic/hivaids/paediatric-treatment-and-care/ (2018).

2. Gunn JKL, Asaolu IO, Center KE, et al. Antenatal care and uptake of HIV testing among pregnant women in sub-Saharan Africa: A cross-sectional study. J Int AIDS Soc. 2016;19. Epub ahead of print. https://doi.org/10.7448/ IAS.19.1.20605.

3. Holmes C, Preko P, Bolds R, et al. Acceptance of voluntary Counselling, testing and treatment for HIV among pregnant women in Kumasi, Ghana. Ghana Med J. 2008:42:8-15.

4. Kizito D, Woodburn PW, Kesande B, et al. Uptake of HIV and syphilis testing of pregnant women and their male partners in a programme for prevention of mother-to-child HIV transmission in Uganda. Trop Med Int Heal. 2008;13:680-2.

5. Tchendjou PT, Koki PN, Eboko F, et al. Factors associated with history of HIV testing among pregnant women and their Partners in Cameroon: baseline data from a behavioral intervention trial (ANRS 12127 Prenahtest). J Acquir Immun Defic Syndr. 2011:57:S9-S15.

6. National Bureau of Statistics (NBS). Tanzania Demographic and Health Survey and Malaria Indicator Survey 2015-2016. 2016

7. UNAIDS. 90-90-90, http://www.unaids.org/en/resources/documents/2017/ 90-90-90 (2017)

8. Negin J, Wariero J, Mutuo P, et al. Feasibility, acceptability and cost of home-based HIV testing in rural Kenya. Tropical Med Int Health. 2009;14: 849-55.

9. Suthar AB, Ford N, Bachanas PJ, et al. Towards universal voluntary HIV testing and counselling: a systematic review and meta-analysis of community-based approaches. PLoS Med. 2013;10:e1001496.

10. Coates TJ, Kulich M, Celentano DD, et al. Effect of community-based voluntary counselling and testing on HIV incidence and social and behavioural outcomes (NIMH project accept; HPTN 043): a clusterrandomised trial. Lancet Glob Heal. 2014;2:e267-77.

11. Parker LA, Jobanputra $K$, Rusike $L$, et al. Feasibility and effectiveness of two community-based HIV testing models in rural Swaziland. Tropical Med Int Health. 2015:20:893-902

12. Sekandi JN, Sempeera H, List J, et al. High acceptance of home-based HIV counseling and testing in an urban community setting in Uganda. BMC Public Health. 2011:11:730

13. Sweat M, Morin S, Celentano D, et al. Community-based intervention to increase HIV testing and case detection in people aged 16-32 years in Tanzania, Zimbabwe, and Thailand (NIMH project accept, HPTN 043): a randomised study. Lancet Infect Dis. 2011;11:525-32.

14. Molesworth AM, Ndhlovu R, Banda E, et al. High accuracy of home-based community rapid HIV testing in rural Malawi. J Acquir Immune Defic Syndr. 2010;55:625-30

15. Kim LH, Arinaitwe E, Nzarubara B, et al. Acceptability and feasibility of serial HIV antibody testing during pregnancy/postpartum and male partner testing in Tororo, Uganda. AIDS Care. 2014;26:360-6.

16. Osoti $\mathrm{AO}$, John-Stewart G, Kiarie JN, et al. Home-based HIV testing for men preferred over clinic-based testing by pregnant women and their male partners, a nested cross-sectional study. BMC Infect Dis. Epub ahead of print. 2015. https://doi.org/10.1186/s12879-015-1053-2.

17. Mark J, Kinuthia J, Roxby A, et al. P3.106 Uptake of home-based point-ofcare syphilis \& hiv testing among male partners of pregnant women inwestern kenya. BMJ Sexually Transmitted Infections Epidemiology, Monitoring and Evaluation. 2017;93:A132-A133.
18. Sharma M, Ying R, Tarr G, et al. Systematic review and meta-analysis of community and facility-based HIV testing to address linkage to care gaps in sub-Saharan Africa. Nature. 2015;528:S77-85.

19. Jatou H, Id C, Mackellar D, et al. Methods, outcomes, and costs of a 2.5 year comprehensive facility-and community-based HIV testing intervention in Bukoba Municipal Council, Tanzania, 2014-2017. Epub ahead of print; 2019. https://doi.org/10.1371/journal.pone.0215654.

20. UNAIDS. 90-90-90 An ambitious treatment target to help end the AIDS epidemic. https://www.unaids.org/en/resources/documents/2017/90-90-90.

21. NBS. Tanzania Demographic and Health Survey and Malaria Indicator Survey 2015-2016. 2016.

22. Adeleke S, Mukhtar-Yola M, Gwarzo G. Awareness and knowledge of mother-to-child transmission of HIV among mothers attending the pediatric HIV clinic, Kano, Nigeria. Ann Afr Med. 2009;8:210.

23. Lyamuya EF, Aboud S, Urassa WK, et al. Evaluation of simple rapid HIV assays and development of national rapid HIV test algorithms in Dar Es Salaam, Tanzania. BMC Infect Dis. 2009:9:19.

24. Munseri PJ, Bakari M, Janabi M, et al. Declining HIV-1 prevalence and incidence among police officers - a potential cohort for HIV vaccine trials, in Dar Es Salaam, Tanzania. BMC Public Health. 2013;13:722.

25. MOHSW. the United Republic of Tanzania Standard Treatment Guidelines and Essential Medicines List Ministry of Health and Social Welfare Fourth Edition. 2016

26. Baltazar CS, Horth R, Inguane C, et al. HIV prevalence and risk behaviors among Mozambicans working in south African mines. AIDS Behav. 2015;19:59-67.

27. Naik R, Doherty $T$, Jackson D, et al. Linkage to care following a home-based HIV counselling and testing intervention in rural South Africa. J Int AIDS Soc. 2015:18:19843.

28. Njau B, Watt MH, Ostermann J, et al. Perceived acceptability of home-based couples voluntary HIV counseling and testing in northern Tanzania. AIDS Care. 2012;24:413-9.

29. Drake AL, Wagner A, Richardson B, et al. Incident HIV during Pregnancy and Postpartum and Risk of Mother-to-Child HIV Transmission: A Systematic Review and Meta-Analysis. PLoS Med. 2014;11. Epub ahead of print. https:// doi.org/10.1371/journal.pmed.1001608.

30. Lawi J, Mirambo MM, Magoma M, et al. Sero-conversion rate of syphilis and HIV among pregnant women attending antenatal clinic in Tanzania: a need for re-screening at delivery. BMC Pregnancy Childbirth. 2015;15:3.

31. Mbizvo MT, Kasule J, Mahomed K, et al. HIV-1 seroconversion incidence following pregnancy and delivery among women seronegative at recruitment in Harare, Zimbabwe Central Afr J Med. 2001:47:115-8.

32. Gray RH, Li X, Kigozi G, et al. Increased risk of incident HIV during pregnancy in Rakai, Uganda: a prospective study. Lancet. 2005:366:1182-8.

33. Thomson KA, Hughes J, Baeten JM, et al. Increased risk of HIV acquisition among women throughout pregnancy and during the postpartum period: a prospective per-coital-act analysis among women with HIV-infected partners. J Infect Dis. 2018;218:16-25.

34. Heemelaar S, Habets N, Makukula Z, et al. Repeat HIV testing during pregnancy and delivery: missed opportunities in a rural district hospital in Zambia. Trop Med Int Heal. 2015;20:277-83.

35. Rogers AJ, Weke E, Kwena Z, et al. Implementation of repeat HIV testing during pregnancy in Kenya: a qualitative study. Epub ahead of print. 2016 https://doi.org/10.1186/s12884-016-0936-6.

36. Lema IA, Sando D, Magesa $L$, et al. Community health workers to improve antenatal care and PMTCT uptake in Dar es Salaam, Tanzania: a quantitative performance evaluation. J Acquir Immune Defic Syndr.2014:67(Suppl 4), S195-S201www.jaids.com. Accessed 16 May 2019

\section{Publisher's Note}

Springer Nature remains neutral with regard to jurisdictional claims in published maps and institutional affiliations. 